\title{
Fire Recognition Based on Multiple Features of Video Images
}

\author{
ZHANG Li-hong \\ School of Physics and Electronic Engineering \\ Shanxi University \\ Tai Yuan, China \\ e-mail:lhzhang@sxu.edu.cn
}

\author{
LIU Xiu-qing \\ School of Physics and Electronic Engineering \\ Shanxi University \\ Tai Yuan, China \\ e-mail: 705048817@qq.com
}

\begin{abstract}
As traditional fire detection techniques have many disadvantages, the fire image automatic recognition algorithm based on video image processing is studied. After the images are preprocessed, multiple fire features will be extracted. Feature extraction has a great influence on fire recognition accuracy. According to video sequence features and the characteristic difference from the flame and other things in background, many flame features such as frame similarity, geometry, color, shape and other features are extracted. Grid searching method is utilized for support vector machine (SVM) to select better parameters. Then these features including frame similarity, red saturation value, area, eccentricity, circularity degree and other features of flame and other interferences are trained by SVM, and finally the fire images can be recognized. The experimental results show that the algorithm is effective, automatic fire detection has higher accuracy and decreases false alarm ratio.
\end{abstract}

Keywords-fire detection; multiple features;feature extraction; support vector machine; image recognition

\section{INTRODUCTION}

Traditional fire detection often employs smoke sensors, temperature sensors, photosensitive detectors, or photoelectric beam detectors. These techniques have some limitations and are subject to conditions such as weather, temperature, places and so on, which contribute to short effective detection distance and small covering range. Fire detection technique based on image processing can effectively overcome the above shortcomings of the conventional detectors, so that long distance and large space fire detection becomes possible. In recent years, people have already started to study fire detection technologies based on image processing deeply. Phillips W[1] used the color and jitter of objects in color video sequences to determine the existence of the flame. Yamagishi[2] put forward a color image flame detection algorithm based on HSV space and neural network, and it can well judge the static image while failing to take advantage of the dynamic characteristics.

In response to these problems, this paper put forward a method for fire video image recognition based on support vector machine (SVM). The characteristic parameters such as eccentricity, color and frame similarity are learned and trained by SVM in the case of a separable mode to identify fire. So a fire can be determined whether it occurs.

\section{PRETREATMENT}

Computational vision image is of relatively poor quality, and there is a lot of noise. Besides, in the scene being monitored, there may be many stationary targets that whose color is similar with flames and smoke. Although they are not noise, their presence severely affect the detection accuracy and system performance. With the size $3 \times 3$ window, median filter and Gaussian filter are used to enhance image. After median and Gaussian filtered, most image noise is effectively inhibited and the image quality is significantly improved. Median filtering is only used within each frame image to eliminate the noise.

\section{FIRE IMAGE FEATURE EXTRACTION}

In order to improve fire recognition accuracy, we choose fire video collection as subjects and select candles, lights, lighters, cigarette butts as disruptors. $15 \mathrm{~s}$ video is captured and 80 images from the $15 \mathrm{~s}$ video sequence are chosen. It is found in the initial stage of the fire that the fire is from scratch and the flame is from small to big. During the process of combustion, the size, shape, location and intensity of radiant energy of the flame are changing [3].This paper selects five typical characteristics for fire identification: frame similarity, red saturation, area value, eccentricity and circularity degree.

\section{A. Frame Similarity}

Similarity measure between adjacent frames of video is as the basis for the initial judgment. There is a high correlation between adjacent frames when no fire occurs. When fire occurs, a sharp difference of similarity values will appear between the fire image and the reference image. With the spread of fire, the image will have more and more different pixels (mainly in the flame region), and frame similarity values will continuously change. Self-correlation function is employed as the similarity between the two adjacent frames.

As the image is $\{f(i, j) ; i, j=0,1,2 \ldots, N-1\}$, then its self-correlation function is:

$$
\rho(x, y)=\frac{\sum_{i=0}^{N-1} \sum_{j=0}^{N-1} f(i, j) f(i+x, j+y)}{\sum_{i=0}^{N-1} \sum_{j=0}^{N-1} f^{2}(i, j)}, \quad \mathrm{x}, \mathrm{y} \geq 0 .
$$


According to the formula (1), the frame similarity between the fire reference frame and the first frame, the second frame and so on will be calculated. Then it will be calculated for candles or lights in accordance with the same sequence of frames. Table 1 is concluded as follow.

TABLE I. FRAME SIMILARITY LIST FOR FIRE IMAGE AND OTHER DISRUPTORS IMAGE

\begin{tabular}{|c|r|r|r|r|r|c|}
\hline & 1 & 2 & 3 & $\ldots$ & 79 & 80 \\
\hline fire & 0.3573 & 0.3216 & 0.3469 & $\ldots$ & 0.3731 & 0.3673 \\
\hline candle & 0.7357 & 0.7369 & 0.7469 & $\ldots$ & 0.7731 & 0.7673 \\
\hline $\begin{array}{c}\text { cigarette } \\
\text { butts }\end{array}$ & 0.9625 & 0.9235 & 0.9635 & $\ldots$ & 0.9478 & 0.9531 \\
\hline headlight & 0.9376 & 0.9269 & 0.9481 & $\ldots$ & 0.9653 & 0.9778 \\
\hline $\begin{array}{c}\text { street } \\
\text { lamp }\end{array}$ & 0.9265 & 0.9634 & 0.9789 & $\ldots$ & 0.9873 & 0.9711 \\
\hline lighter & 0.6016 & 0.6346 & 0.6244 & $\ldots$ & 0.6648 & 0.6518 \\
\hline
\end{tabular}

Table 1 shows that frame similarity of candles, lights, and cigarette butts and so on is larger than that of fire video sequence. It is because that the shape and the location of the flame changes sharply in the combustion process.

\section{B. Red Saturation}

Generally from the flame core to the outer flame, the fire color changes from white to red and the process is gradual.The color of the whole flame is often one or a few colors.

With color feature, flame and objects that have the color similar to flame can be distinguished effectively because the color of headlight, cigarette butts and so on is mostly monochrome. These objects don't have a color distribution feature flame has. According to this, recognition algorithm is put forward as follow.

$$
\text { redratio }(x, y)=\sum_{x, y \in m} \frac{R\left[P_{i}(x, y)\right]}{R\left[P_{i}(x, y)\right]+G\left[P_{i}(x, y)\right]+B\left[P_{i}(x, y)\right]}
$$

In the formula (2), $P_{i}$ is the ith frame of continuous $n$ frame images. R, G, B are separately the number of pixels of red, green, and blue color in the image. A sequence of continuous 100 early fire images and disruptors are selected, and then the red saturation is calculated. Concrete experimental data are in the table 2 as follow.

TABLE II. RED RATIO LIST OF FIRE IMAGE AND OTHER DISRUPTORS

\begin{tabular}{|c|c|c|c|c|c|c|}
\hline & 1 & 2 & 3 & $\ldots$ & 79 & 80 \\
\hline \multicolumn{1}{|c|}{ fire } & 0.5727 & 0.6013 & 0.6014 & $\ldots$ & 0.6471 & 0.7010 \\
\hline candle & 0.2911 & 0.2986 & 0.2731 & $\ldots$ & 0.2912 & 0.2813 \\
\hline $\begin{array}{c}\text { cigarette } \\
\text { butts }\end{array}$ & 0.2031 & 0.2140 & 0.2011 & $\ldots$ & 0.2097 & 0.2083 \\
\hline headlight & 0.3421 & 0.2742 & 0.3711 & $\ldots$ & 0.2989 & 0.4772 \\
\hline $\begin{array}{c}\text { street } \\
\text { lamp }\end{array}$ & 0.1243 & 0.1317 & 0.2032 & & 0.2096 & 0.2083 \\
\hline lighter & 0.2779 & 0.2813 & 0.2910 & & 0.2972 & 0.2053 \\
\hline
\end{tabular}

Table 2 shows that the red ratio of the candle flame, cigarette butts and lighter image do not have significant changing rule while it presents irregular bounce, but headlight is similar to fire flame, its red ratio presents a continuous growth trend. Therefore it is needed to combine with other image characteristics to judge.

\section{Area Value}

The change of the flame area evaluates the area changing characteristics in the process of fire. In the process of fire, flame area changes. Most interference objects' area changing value is small [4]. E.g. moving car light's area is almost the same although coordinate position changes all the time. Flame area value of the continuous frame is calculated and employed as one of fire characteristics. Usually the area of the flame can be expressed by counting the number of pixels included in the area. By the image segmentation flame area is divided out, and binary area obtained by the division is the target area. Then the area value of the target area can be obtained by counting the number of pixel points whose pixel value is 1 . The flame area pixels are marked as $f(m, n)=1$, background as $\mathrm{f}(\mathrm{m}, \mathrm{n})=0$. Then the flame area will be marked as:

$$
S=\sum_{i=0}^{m-1} \sum_{j=0}^{n-1} f(i, j) .
$$

Continuous 100 early fire frame images and video sequences of the interference objects are chosen, then the area value of flame in each frame or lights in interference objects are calculated, and the specific experimental data are shown in table 3.

TABLE III. AREA VALUE LIST OF FLAME IMAGE AND OTHER INTERFERENCE IMAGE S

\begin{tabular}{|c|l|l|l|l|l|l|}
\hline & 1 & \multicolumn{1}{|c|}{2} & 3 & $\ldots$ & 79 & 80 \\
\hline fire & 1291 & 1756 & 2069 & $\ldots$ & 2335 & 2385 \\
\hline candle & 389 & 402 & 388 & $\ldots$ & 406 & 403 \\
\hline $\begin{array}{c}\text { cigarette } \\
\text { butts }\end{array}$ & 98 & 101 & 97 & $\ldots$ & 105 & 99 \\
\hline headlight & 129 & 158 & 231 & $\ldots$ & 376 & 423 \\
\hline street lamp & 410 & 408 & 411 & $\ldots$ & 423 & 409 \\
\hline lighter & 389 & 402 & 388 & $\ldots$ & 406 & 403 \\
\hline
\end{tabular}

Table 3 shows that flame area value changes sharply while the area of interfering objects whose color is similar to fire fluctuate almost in a small certain value.

\section{Eccentricity Ratio}

Eccentricity ratio, also called elongation, is used to distinguish between elongated objects and approximately regular interference objects [3].The shape of flame in the combustion process is not fixed, sometimes it presents slender form, sometimes flat wide type, while the interference area rarely shows such shapes. So eccentricity ratio can be used to represent the difference between flame area and interference objects (candles, light, etc.) .The calculation formula of the eccentricity ratio is:

$$
T=\min (l, w) / \max (l, w) .
$$

In the formula (4), 1, w respectively stands for width and height of the prospect.

Continuous 100 early frame fire images and video sequences of the interference objects are chosen, and the 
eccentricity ratio is calculated. The specific experimental data are shown in table 4.

TABLE IV. ECCENTRICITY RATIO LIST OF THE FIRE IMAGE AND OTHER INTERFERENCE IMAGES

\begin{tabular}{|c|r|r|r|r|r|r|}
\hline & 1 & 2 & 3 & & 79 & 80 \\
\hline fire & 1.5178 & 1.5179 & 1.3787 & $\ldots$ & 1.1993 & 1.4973 \\
\hline candle & 3.3880 & 3.2614 & 3.5122 & & 3.4167 & 3.5500 \\
\hline $\begin{array}{c}\text { cigarette } \\
\text { butts }\end{array}$ & 1.1096 & 1.0959 & 1.1111 & & 1.1096 & 1.1486 \\
\hline headlight & 1.2093 & 1.2347 & 1.3100 & & 1.2370 & 1.3206 \\
\hline $\begin{array}{c}\text { street } \\
\text { lamp }\end{array}$ & 2.9510 & 2.9510 & 2.9510 & & 2.9900 & 3.0700 \\
\hline lighter & 3.7818 & 3.5690 & 4.0000 & & 3.2344 & 3.8000 \\
\hline
\end{tabular}

Table 4 shows that the eccentricity ratios of interference objects are larger than that of the flame images because cigarette butts, car light, street lamp are of round shape, regular shape or approximately equal height and width.

\section{E. Degree of Circularity}

Degree of circularity can measure shape of an object [7]. Fire flame's shape is irregular, and most interference sources (street lamp, candle flame, etc.) are of approximately regular shapes. Therefore flame circularity degree may be used as a criterion for fire recognition. The calculation formula of the degree of circularity is:

$$
C_{k}=\frac{L_{k}^{2}}{4 \pi S_{k}} \text {. }
$$

In the formula (5), $C_{k}$ stands for the degree of circularity of the kth image, $L_{k}$ for the circumference of the kth image's prospect, $S_{k}$ for the area of the kth image prospect.

Continuous 100 early frame fire images and sequences of the interference objects video are chosen, then the degree of circularity is calculated. The specific experimental data are shown in table 5.

TABLE V. CIRCULARITY DEGREE LIST OF THE FIRE IMAGE AND OTHER INTERFERENCE IMAGES

\begin{tabular}{|c|l|l|l|l|l|l|}
\hline & \multicolumn{1}{|c|}{1} & \multicolumn{1}{|c|}{3} & \multicolumn{1}{|c|}{3} & & \multicolumn{1}{|c|}{79} & 80 \\
\hline fire & 9.7041 & 9.9401 & $\begin{array}{l}10.413 \\
6\end{array}$ & & $\begin{array}{l}10.711 \\
9\end{array}$ & $\begin{array}{l}10.247 \\
6\end{array}$ \\
\hline candle & 2.6041 & 2.3342 & 2.5281 & & 2.7510 & 2.7092 \\
\hline $\begin{array}{c}\text { cigarette } \\
\text { butts }\end{array}$ & 1.5830 & 1.3746 & 1.4221 & & 1.5446 & 1.0114 \\
\hline headlight & 1.0992 & 1.2093 & 1.2179 & & 1.2843 & 1.2008 \\
\hline $\begin{array}{c}\text { Street } \\
\text { lamp }\end{array}$ & 1.2093 & 1.2179 & 1.1358 & & 1.2008 & 1.2309 \\
\hline lighter & 1.5830 & 1.3746 & 1.4221 & & 1.5446 & 1.0114 \\
\hline
\end{tabular}

Table 5 shows that the circularity degree of flame is bigger than that of interference objects such as cigarette butts, lighters.

\section{SUPPORT VECTOR MACHINE}

Support Vector Machine (SVM) is firstly proposed by Vapnik. Like a multilayer perceptron network and radial basis function network, it can be used for pattern classification and nonlinear regression. Its main idea is to establish a classification hyperplane as a decision curved surface to maximize the separating edge between the positive and negative examples $[4,5]$. SVM is based on the statistical theory, more precisely, it is a approximate realization of structural risk minimization.

In order to set up fire model, firstly the training set and test set are extracted from the original characteristics data, then the data are pre-processed, and SVM is employed for training the training set. Finally the model is utilized to predict category labels of the test set and the accuracy of the recognition of the fire can obtained. The algorithm process is shown in Fig. 1.

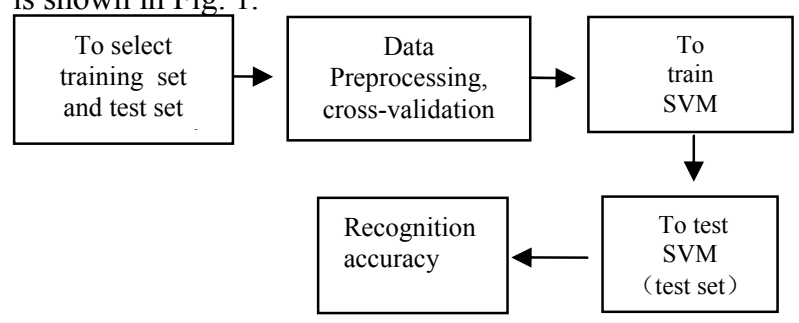

Figure 1. Procedure of fire recognition modle

\section{A. Select training Set and Test Set}

For training set [6]:

$$
T=\left\{\left(x_{1}, y\right),\left(x_{2}, y\right) \cdots\left(x_{l}, y\right)\right\} .
$$

$\mathrm{X}$ is training samples, $\mathrm{y}$ is the label of training samples. In order to establish the decision function:

$$
f(x)=\operatorname{sgn}\left\{\sum_{i=1}^{l} a_{i}^{*} y_{i} K\left(x_{i}, x\right)+b^{*}\right\} \text {. }
$$

$A$ and $b$ in the formula (7) can be obtained by constructing and solving optimization problems ( 8) .

$$
\begin{array}{ll}
\min & f(a)=\frac{1}{2} \sum_{i, j=1}^{l} a_{i} a_{j} y_{i} y_{j}\left(K\left(x_{i}, x_{j}\right)\right)-\sum_{i=1}^{l} a_{i} \\
\text { s.t. } & \left\{\begin{array}{l}
\sum_{i=1}^{n} y_{i} a_{i}=0 \\
0 \leq a_{i} \leq C, i=1,2, \cdots l
\end{array}\right.
\end{array}
$$

The optimal solution is:

$a^{*}=\left(a_{1}^{*}, a_{2}^{*}, \cdots a_{l}^{*}\right)^{T}$, choose $0<a_{j}^{*}<C$.

Then the threshold is calculated according to this:

$$
b^{*}=y_{j}-\sum_{i=1}^{l} y_{i} a_{i}^{*} K\left(x_{i}, x_{j}\right) \text {. }
$$

$\mathrm{C}$ in the formula is penalty function, $\mathrm{K}\left(\mathrm{x}_{\mathrm{i}}, \mathrm{x}_{\mathrm{j}}\right)$ is kernel function, the common kernel functions are as follows[5]:

- Polynomial kernel function:

$$
K(x, y)=(x \cdot y+1)^{d}, \mathrm{~d}=1,2, \ldots \ldots
$$

- Radial basis kernel function:

$$
K(x, y)=\exp \left(-\gamma\|x-y\|^{2}\right)
$$

- $\quad$ Sigmoid kernel function :

$$
K(x, y)=\tanh (b(x \cdot y)-c)
$$




\section{B. Data Pre-processing}

Before the training set and test set are trained and tested, data normalization must be done. This can improve the classification accuracy. But different normalization methods have different influence on the final accuracy.

A normalization mapping is as follows to normalize the training set and test set $[6,7]$.

$$
f: x \rightarrow y=\frac{x-x_{\text {min }}}{x_{\text {max }}-x_{\min }}
$$

In the formula (10) $, \quad x, y \in \mathrm{R}^{\mathrm{n}}, \mathrm{x}_{\min }=\min (\mathrm{x})$, $\mathrm{x}_{\max }=\max (\mathrm{x})$. Normalization effect is that the original data is structured to $[0,1]$ range. That means $\mathrm{y}_{\mathrm{i}} \in[0,1], \mathrm{i}=1,2 \ldots, \mathrm{n}$, This is called $[0,1]$ interval normalization.

\section{Training and Testing}

Select 480 image frames as the samples for experiments, in which 260 image frames are fire video samples, 320 frames are no fire samples. No fire samples are randomly inserted into the fire samples. The characteristics data of 1350 images are as training samples, feature data of 351-480 images are as test samples.

\section{THE EXPERIMENTAL DATA AND SIMULATION}

In this sample experiment 480 frame fire and interference images are divided into two types of samples, 1-350 frame samples are trained to obtain model, another 351-480 for testing. Radial basis function is utilized as kernel function for SVM. The image features data are shown in Table 6.

TABLE VI. FEATURE DATA OF FLAME AND INTERFERENCE IMAGES

\begin{tabular}{|l|c|c|c|c|c|c|}
\hline & $\begin{array}{c}\text { Circularity } \\
\text { degree }\end{array}$ & $\begin{array}{c}\text { area } \\
\text { value }\end{array}$ & $\begin{array}{c}\text { Eccentricity } \\
\text { ratio }\end{array}$ & $\begin{array}{c}\text { red } \\
\text { ratio }\end{array}$ & $\begin{array}{c}\text { Frame } \\
\text { similarity }\end{array}$ & Label \\
\hline 1 & 2.6041 & 389 & 3.3880 & 0.2991 & 0.3571 & 0 \\
\hline 2 & 2.0982 & 345 & 3.7818 & 0.2779 & 0.6016 & 0 \\
\hline 3 & 1.5830 & 98 & 1.1096 & 0.2031 & 0.9625 & 0 \\
\hline 4 & 9.7041 & 1291 & 1.5178 & 0.5727 & 0.3573 & 1 \\
\hline$\ldots$ & $\ldots \ldots$ & $\ldots \ldots$ & $\ldots \ldots$ & $\ldots \ldots$ & $\ldots \ldots$ & $\ldots \ldots$ \\
\hline 350 & 9.4901 & 1916 & 1.1517 & 0.6041 & 0.3469 & 1 \\
\hline 351 & 1.2843 & 419 & 2.9608 & 0.2096 & 0.9164 & 0 \\
\hline 352 & 1.2008 & 423 & 3.0700 & 0.2083 & 0.9711 & 0 \\
\hline$\ldots$ & $\ldots \ldots$ & $\ldots \ldots$ & $\ldots \ldots$ & $\ldots \ldots$ & $\ldots \ldots$ & $\ldots \ldots$ \\
\hline 480 & 10.2476 & 2385 & 1.4937 & 0.7010 & 0.3673 & 1 \\
\hline
\end{tabular}

Then the flame and the interference image feature data in the table VI are normalized later.

In order to make SVM reach the optimal classification, the training set must be utilized to find better parameters for classification, so that the test set accuracy is maintained at a higher level. In order to obtain optimal parameters (c, g ), this paper selects the grid searching method to find the optimal parameters $(\mathrm{c}, \mathrm{g})$. Log2c and $\log 2 \mathrm{~g}$ value range is 10 to 10 . The best accuracy often corresponds with a plurality of groups of $\mathrm{c}$ and $\mathrm{g}$. Then in these parameters which can achieve optimal accuracy, the smallest $\mathrm{c}$ group will be chosen.
TABLE VII. RECOGNITION RESULTS AFTER SIMULATION

\begin{tabular}{|l|l|l|}
\hline & \multicolumn{1}{|c|}{ Accuracy } & \multicolumn{1}{|c|}{ Parameters options } \\
\hline Not to be normalized & $60 \%(288 / 480)$ & best c=2,best $\mathrm{g}=1$ \\
\hline$[0,1]$ normalization & $87.5 \%(420 / 480)$ & best $\mathrm{c}=2$, best $\mathrm{g}=1$ \\
\hline Grid search & $97 \%(466 / 480)$ & $\begin{array}{l}\text { Best } \mathrm{c}=0.25, \text { best } \\
\mathrm{g}=0.75786\end{array}$ \\
\hline
\end{tabular}

The experimental results show that, before training and testing, normalizing data set can improve recognition accuracy and the choice of the optimal parameters (c, g ) can obtain better accuracy. Too high parameter $\mathrm{c}$ easily leads to overlearning state. So we select the smallest parameter group in all parameters (c, g) which can achieve the highest accuracy of verification. And the grid searching method employs this parameter selection principle, and the fire recognition ratio can reach $97 \%$.

\section{CONCLUSION}

Based on support vector machine, with multiple features of video images, fire image recognition method can accurately identify the fire images. It is a kind of new fire monitoring method which overcomes the disadvantages of the traditional fire detection technology affected by environmental factors, so that the fire monitoring system is of a self-organizing and adaptive function and it has a broad market prospect.

\section{ACKNOWLEDGMENT}

This work is supported by Scientific and Technological Innovation Programs of Higher Education Institutions in Shanxi province (2010002). The authors also would like to express their gratitude to the other members of the algorithm team for valuable discussions.

\section{REFERENCE}

[1] W. Phillips. Flame recognition in video. Fifth IEEE Workshop on Applications of Computer Vision. California.2000.pp.224-229.

[2] YAMAGISHI H,YAMAGUCHI J. Fire flame detection algorithm using a color camers[J]. Proc.1999 International Symposium on Micromechatronics and Human Science. 1999.pp.255-260.

[3] Yuan Feiniu, Liao Guangxuan, Zhang Yongming. Fire detection feature extraction based on computer vision [J]. Journal of University of Science \& Technology China, 2006, 36 ( 1).pp. 39-43.

[4] Huaiqin Qi, Xu Gang, Wang Na. Forest Fire Recognition Algorithm Based on Video [J]. Video Applications and Engineering. Vol. 34, 2010 .pp.116-118

[5] Yang Zhimin, Liu Guangli. The Uncertainty Principle of Support Vector Machine and Its Application [M]. Science Press.2007,4

[6] Turgay Celik, Kai-kuang Ma. Computer Vision Based Fire Detection in Color Images. Pattern Recognition.2008,34(12).pp.258263.

[7] Yu-Chiang Li. Sequential Pattern Technology for Visual Fire Detection. Journal of Electronic Science and Technology.2012,3. pp.70-74 